\title{
El estado de la guitarra eléctrica en la música académica contemporánea
}

\section{Artículo de reflexión}

\section{Johan León Ramírez}

Círculo Colombiano de Música Contemporánea, CCMC johanleonemail@gmail.com

Recibido: 24 de marzo de 2017

Aprobado: 10 de mayo de 2017

Cómo citar este artículo: León Johan. (2018) El estado de la guitarra eléctrica en la música académica contemporánea. Calle14: revista de investigación en el campo del arte 13 (23) pp. 38-53. DOI: https://doi. org/10.14483/21450706.12988 


\section{Resumen}

El objetivo de este escrito es dar importancia al repertorio para guitarra eléctrica comprendido en la producción de música académica contemporánea y, al mismo tiempo, proporcionar valor al instrumento dentro de esta estética musical. Para esto, se hace un estudio de los antecedentes relacionados con el tema tratado, examinando algunos textos escritos por otros autores con preocupaciones similares a las mías, observando posteriormente el trabajo artístico que robustece el repertorio existente, referenciando el trabajo realizado por algunos ensambles, guitarristas $\mathrm{y}$, específicamente, compositores en Colombia.

\section{Palabras claves}

Composiciones, compositores, guitarra eléctrica, guitarristas, interpretación, música académica contemporánea.

\section{The status of the electric guitar in gontemporary academic music}

\section{Abstract}

The aim of this paper is to give importance to the repertoire of electric guitar found in contemporary academic music and, at the same time, to provide value to the instrument within this musical aesthetics. For this, the antecedents of the matter are examined, reviewing texts written by other authors with similar concerns to mine; we take a closer look at the artistic work that strengthens the existent repertoire, and, finally we reference the work done by some ensembles, guitarists and, specifically, composers in Colombia.

\section{Keywords}

Compositions, composers, electric guitar, guitarists, interpretation, contemporary academic music.

Le statut de la guitare électrique dans la musique académique contemporaine

\section{Résumé}

Le but de cet article est de donner de l'importance au répertoire de la guitare électrique dans la musique académique contemporaine et, en même temps, de donner de la valeur à l'instrument dans cette esthétique musicale. Pour cela, les antécédents de la question sont examinés, en face de textes écrits par d'autres auteurs avec des préoccupations similaires à la mienne ; nous regardons de plus près le travail artistique qui renforce le répertoire existant, et, enfin, nous référons au travail effectué par certains ensembles, guitaristes et, en particulier, compositeurs en Colombie.

\section{Mots clés}

Compositions, compositeurs, guitare électrique, guitaristes, interprétation, musique académique contemporaine.

\section{O status da guitarra elétrica na música acadêmica contemporânea}

\section{Resumo}

O objetivo deste artigo é dar importância ao repertório de fontes de guitarra elétrica na música acadêmica contemporânea e, ao mesmo tempo, proporcionar valor ao instrumento dentro desta estética musical. Para isso, os antecedentes do assunto são examinados, revisando textos escritos por outros autores com preocupações semelhantes às minhas. Examinamos de perto o traba- 
Iho artístico que fortalece o repertório existente e, finalmente, fazemos referência ao trabalho realizado por alguns conjuntos, guitarristas e, especificamente, compositores na Colômbia.

\section{Palavras-chave}

Composições, compositores, guitarra elétrica, guitarristas, interpretação, música acadêmica contemporânea.

Kai punchakuna sug runa uichiskamanda suma tunaikunawa

\section{Maillallachiska:}

Kai kimi iachangapa imasami tunarka sug wagki guitarawa chasa iachingapa chasaiatata i kawachingapa imawantami niria suma kagmanda kai tunai. Chasami kai iachaikuikuna, iachi kawaspa, chiwanka kai iachaikuikuna kami chasaiatata rigcha kai iachachaikuna, ikuti kai iachaikunata kami kai iachaikuikuna kami ruraska sug runa tunagkunawa i kai kami ruraska kai suti alpa kutumbiaspi.

\section{Rimangapa Ministidukuna:}

Ruraikuna, ruragkuna, tunagkuna, uichagkuna kunaurakuna. 


\section{Aclaraciones preliminares}

Pese a que el objetivo principal de este texto no es problematizar un hecho estético, se hace importante precisar a qué me refiero con Música Académica Contemporánea, para los fines específicos del escrito:

se entiende por Música Académica

Contemporánea al repertorio creado a partir de la segunda mitad del siglo XX, vinculado a la tradición musical académica (también conocida como clásica, erudita, docta, culta, de arte o de concierto). Esto incluye música acústica, mixta (medios acústicos y electrónicos), géneros como la música experimental, el arte sonoro y su empleo para otros campos artísticos como la danza, el arte audiovisual, el teatro y otros. (Círculo Colombiano de Música Contemporánea, 2010: 1)

A su vez, este texto es muestra de la investigación hecha en el tema durante los últimos semestres de la carrera de Artes Musicales ofertada por la Facultad de Artes ASAB de la Universidad Francisco José de Caldas en Bogotá, Colombia.

\section{Hipótesis}

Existe el material suficiente que se ocupa del tema de la guitarra eléctrica dentro de la música académica contemporánea como para que sea ampliado el espectro estético al cual se encuentra históricamente condenado el instrumento.

\section{Introducción}

Innegablemente, la guitarra eléctrica está ligada a las músicas populares estadounidenses y gran parte de los desarrollos se hicieron conocidos gracias a la popularidad y constante promoción de estas. Sin embargo, de forma paralela, se apropió la guitarra eléctrica en las prácticas musicales académicas contemporáneas.

\section{Referentes}

Contemporary Compositional Techniques for the Electric Guitar in United States Concert Music escrito por el compositor, director y guitarrista eléctrico Robert Tomaro, hace referencia a la composición para la guitarra eléctrica en la música para concierto estadounidense de 1960 a 1994. En este escrito se tienen en cuenta cinco obras (String Trio (1960), Sonar Plexus (1968), Songs, Drones and Refrains of Death (1968), Dirge (1983) y Vicious Cycle (1984)), las cuales se analizan desde cuatro perspectivas que buscan evidenciar el desarrollo y el impacto de la guitarra eléctrica dentro de la música contemporánea. Dichas ideas son:

El desarrollo de la práctica interpretativa de la guitarra eléctrica en la música de concierto estadounidense y su impacto en las técnicas de composición a lo largo del periodo de tiempo entre 1960 y 1994.

El uso de la guitarra eléctrica como un símbolo de un aspecto de la cultura moderna estadounidense. Las diferentes aproximaciones compositivas y maneras en las cuales la composición para la guitarra eléctrica cambia a medida que nuevas técnicas se hacen disponibles.

La exploración de piezas que representen el punto de vista cultural y psicológico cambiante del compositor de música de concierto estadounidense, el cual elige componer para la guitarra eléctrica.

String Trio (1960), para guitarra eléctrica, violín y violonchelo, escrita por el compositor Donald Erb (E.U., 1927 - 2008) se convierte en el punto base para el análisis y comparación de las obras.

A la luz de los puntos más importantes de este estudio se resalta que fue creada a manera de experimento, el cual busca evidenciar la manera en que artistas de diferentes tradiciones musicales (un guitarrista de Jazz y dos músicos con formación en música clásica) funcionarían en una situación de ensamble de cámara. Por otro lado, el autor puntualiza diferentes aspectos formales y técnicos en los que se destaca la escritura con notación estándar, la construcción de la obra basada en glissandos como motivos melódicos (siendo un punto importante debido a la gran diferencia en cómo funciona esta técnica en la guitarra eléctrica y en los instrumentos de cuerda frotada) y la limitación tanto en el rango dinámico como en las capacidades de articulación de la guitarra eléctrica.

Pese a que no existe en este texto un motivo claro por el cual el autor eligió este punto de partida, la presentación de esta primera pieza me permite inferir que parte de la música de concierto estadounidense para guitarra eléctrica está nutrida de diferentes prácticas 


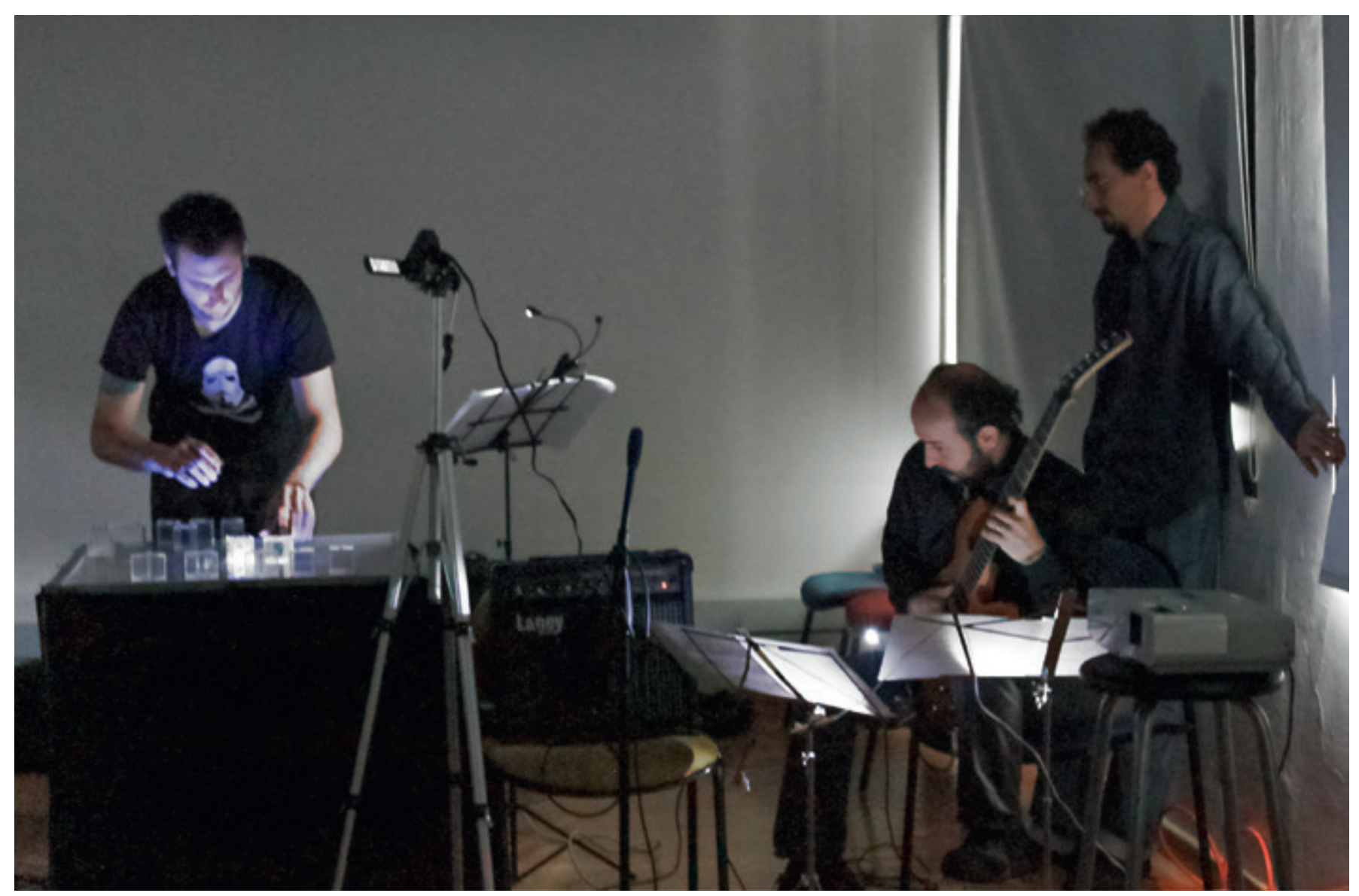

Estreno de la obra: En la cercanía, en cada centímetro de piel... en cada poro (2011) del compositor Luis Fernando Sánchez (Col., 1982), Universidad de los Andes, salón TX-201, 26 de Marzo de 2012. Foto: Alexa Ospina. En la foto de izquierda a derecha: Jürgen Echeverri, Guillermo Bocanegra, Luis Fernando Sánchez.

de la música tradicional en la que esta se desempeña, sin embargo con el análisis de una sola obra no podría asegurarse que todo este repertorio funcione de esta manera, principalmente desconociendo el desarrollo paralelo del instrumento, tanto en la música popular y la música académica. Por otro lado, refiriéndome al análisis de Tomaro, se muestra de forma somera la inclusión de un sonido Jazz dentro de la obra de Erb. Para aclarar esta última afirmación planteo los siguientes cuestionamientos, los cuales no son aclarados en el escrito: ¿Qué elementos dentro de la composición son referencia directa a esta tradición, además del simple uso de la guitarra eléctrica? ¿Qué especificaciones técnicas extraídas de la tradición Jazz tiene la composición?, o ¿son estos elementos, como la limitación en las formas de articulación, únicamente incluidos en la obra por el intérprete como resultado de su experiencia?

La pieza que se ubica cronológicamente en el segundo lugar del análisis es Sonar Plexus (1968) de Francis Thorne (E.U., 1922) escrita para orquesta y guitarra eléctrica. La obra muestra una faceta del instrumento fundamentada en la estética del rock and roll. En esta se incluye una amplia modificación del sonido mediante el uso de distorsión e implementa variadas técnicas de articulación. La guitarra eléctrica mantiene un proceso de aceleración y aumento dinámico constante a través de la composición. Paralelamente a la descripción de la pieza desde una vista panorámica se realiza una comparación con la obra de Erb, haciéndose evidente que ambos compositores hacen uso de un registro medio limitado en la guitarra eléctrica que genera tensión tonal.

Con esta composición Tomaro cambia claramente de foco estético, no obstante, surgen los mismos cuestionamientos anteriores que no son aclarados. Sin embargo, se hace importante el uso de distorsión, aunque el tema se toque de manera superficial.

En este caso el instrumento simboliza las estéticas y tecnologías del siglo $X X$ al usar un amplio rango de modificación sonora y variadas técnicas de articulación, que no se especifican. Debido a esto surgen las 


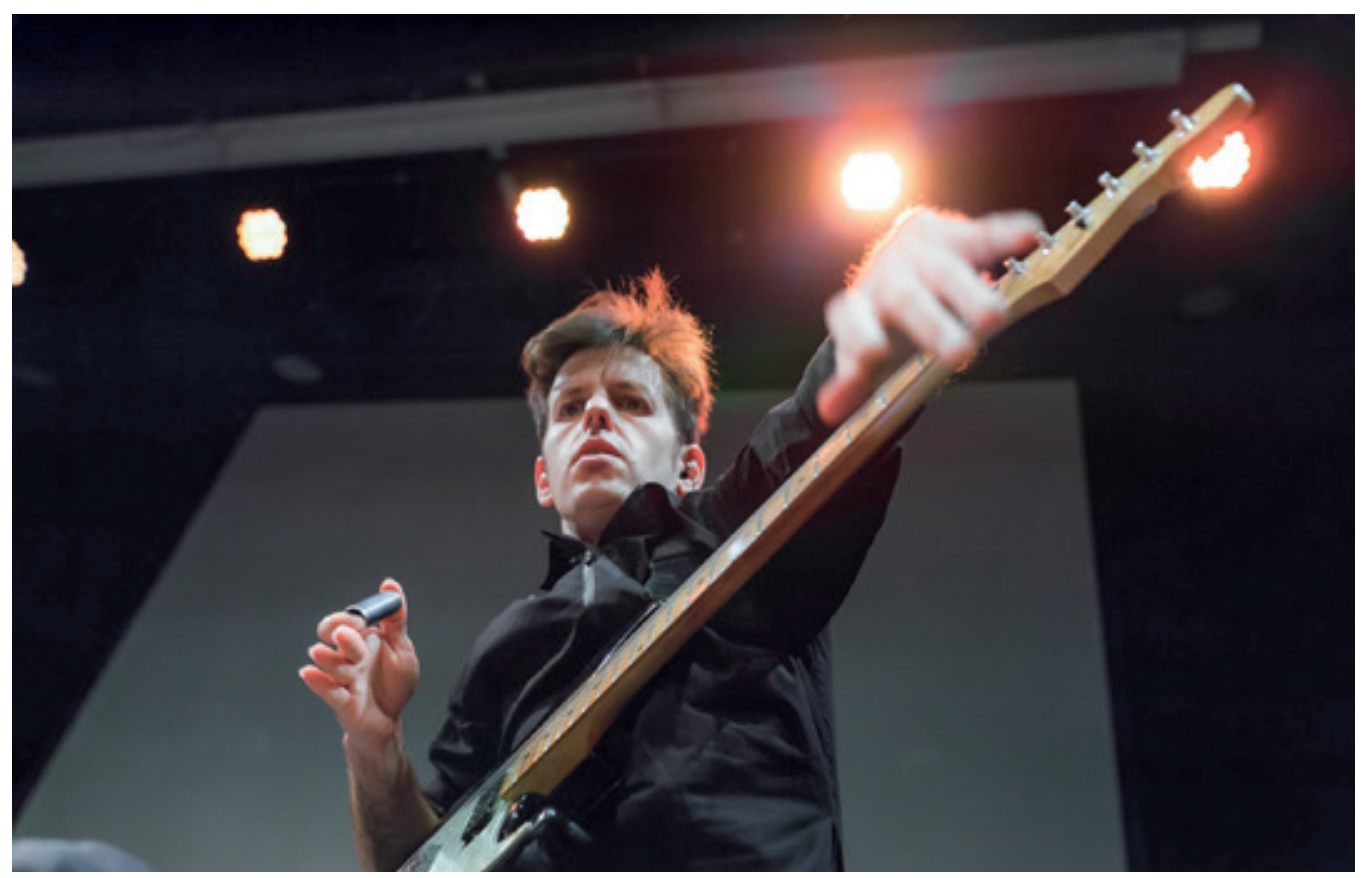

Ensemble Nikel. Foto: Markus Sepperer. En la foto: Yaron Deutsch.

preguntas ¿Qué tipos de modificaciones sonoras son usadas específicamente? ¿Son las técnicas de articulación consecuencia de estas modificaciones? ¿Son tanto las modificaciones sonoras como las técnicas de articulación sugeridas y puestas a libre interpretación del intérprete? 0, por el contrario, ¿son especificadas minuciosamente por el compositor?

El autor insiste en las similitudes entre las obras hasta ahora analizadas, haciendo énfasis en el uso del registro medio de la guitarra, por lo que surge la siguiente pregunta: ¿Se podría afirmar que existe una preferencia al uso de cierta tesitura en el instrumento?, y si sí, ¿es heredada de otra tradición musical o está esto ligado estrechamente a la instrumentación específica de la composición y al rol que le asigna cada compositor al instrumento?

Otra composición abordada por Tomaro es Songs, Drones and Refrains of Death (1968), del compositor George Crumb (E.U., 1929), para ensamble de guitarra eléctrica, bajo eléctrico, clavecín eléctrico, piano amplificado, percusión y barítono. En ella Crumb adapta cuatro poemas de Federico García Lorca. Para esta creación musical el instrumentista hace uso de un slide de vidrio derivado de la música blues. El autor etiqueta la obra de influyente en el desarrollo de técnicas de articulación extendidas en la guitarra eléctrica, haciendo uso de notación sin métrica y ajustada en patrones circulares. A diferencia de las dos composiciones analizadas anteriormente, en Songs, Drones and Refrains of Death se alterna entre los registros más grave y más agudo de la guitarra, usando técnicas como trinos, glissandi y armónicos.

En esta instancia del texto, si bien se especifica un poco más acerca de la obra, sigue sin ser suficiente la información que se proporciona. Es importante la referencia que se hace a la forma en que es tañida la guitarra usando un slide de vidrio. Sin embargo, un aporte valioso habría sido precisar ciertos detalles como: ¿Es usado el slide de igual forma como se usa en el blues o se hace de forma diferente sin guardar más relación con esta música que los referentes históricos del instrumento dentro de ella? Por otro lado, pese a que se hace mención a la relevancia de esta pieza en el desarrollo en las técnicas de articulación, no se hace mención especial acerca de alguna de ellas.

Vale la pena resaltar que esta composición sitúa la guitarra eléctrica junto a una instrumentación que no tiene raíz en ninguna otra música popular estadounidense, aspecto que puede llegar a influenciar drásticamente el tratamiento que se dé al material sonoro que asigne el compositor al rol de la guitarra. Es probable que, por este motivo, en esta obra se hace uso del registro más alto y más bajo de la guitarra en afinación estándar (no se profundiza en este aspecto). 


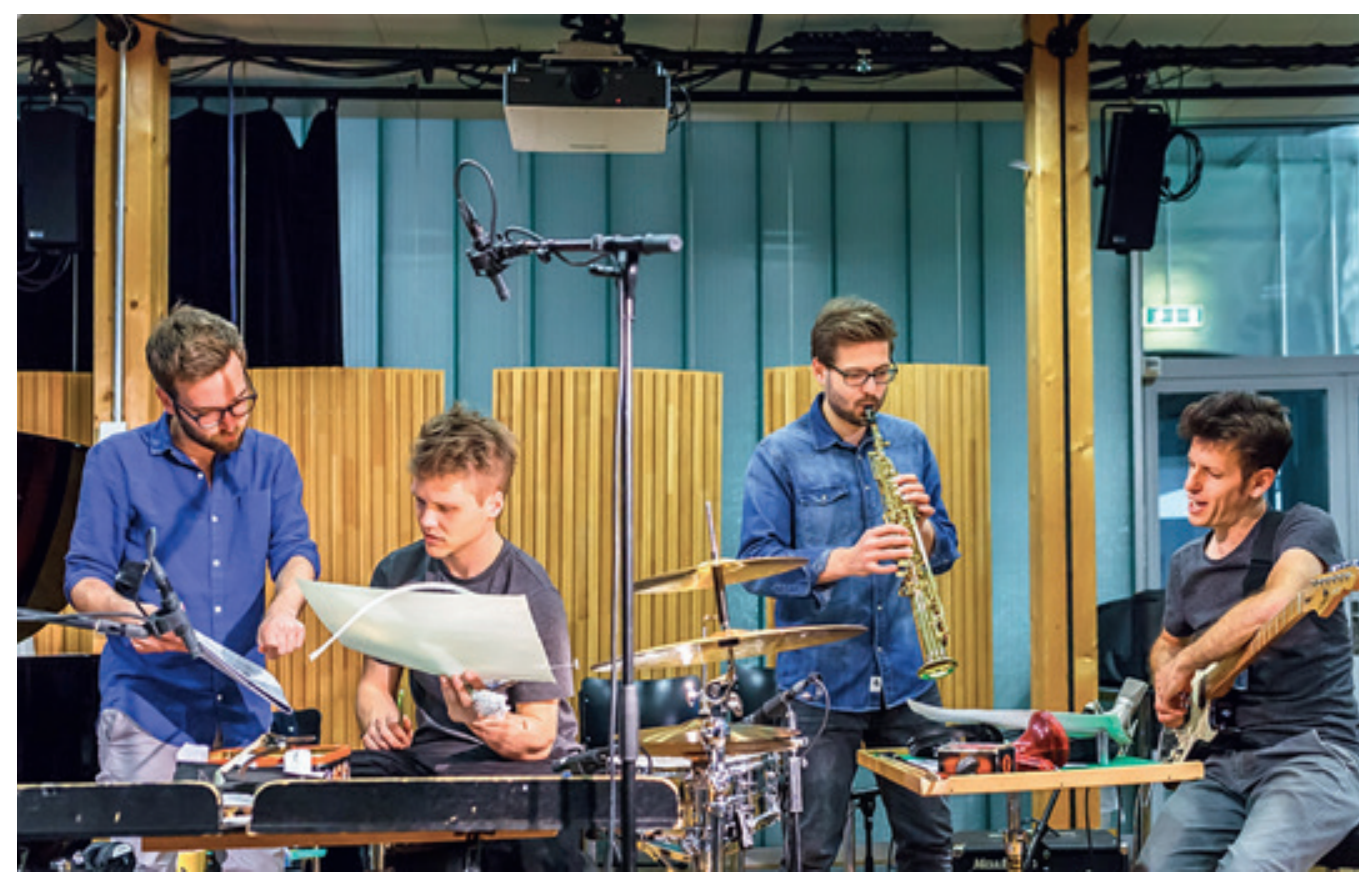

Ensemble Nikel. Foto: Markus Sepperer. En la foto de izquierda a derecha: Antoine Françoise, Brian Archinal, Patrick Stadler, Yaron Deutsch.

Situada quince años después de la pieza anterior, Dirge (1983), del compositor Jeffrey Lohn (E.U.) y escrita para ensamble de guitarras eléctricas, se aleja más, desde un punto de vista instrumental, de los contextos del blues y el rock. Pese a lo anterior Tomaro asegura lo contrario, hecho que podría deberse, en realidad, a la sonoridad misma de la obra. El uso de guitarras eléctricas con distorsión, volumen extremo, técnicas de articulación inusual, como nuevas técnicas de trémolo, y actividad frecuencial en masa son sus características expuestas en el texto. La forma es modificada por el director a medida que transcurre la pieza gracias a que el compositor utiliza una notación simplemente como diseño global que se transforma al ser interpretado.

Nuevamente los detalles son bastante escasos. Tomaro no menciona qué hace que estas formas de articulación sean nuevas, ni qué otro tipo de técnicas se usan en la pieza. De manera semejante, no se aclara el tratamiento que se da a la distorsión desde la perspectiva de la notación y del manejo de parámetros específicos. En cuanto la obra en general, surge el siguiente cuestionamiento: ¿Existe algún otro medio electrónico adicional a la distorsión o alguna disposición espacial específica que apoye los temas referentes a la forma o la percepción de la pieza?

Como composición de cierre se encuentra Vicious Cycle (1984), escrita por Elliot Sharp (E.U., 1951), compuesta para guitarra eléctrica, bajo eléctrico y percusión, lo que propone un ambiente común para el instrumento. La guitarra hace uso de un sonido modificado de manera única, con diferentes técnicas de articulación y scordatura. En un plano general, la obra hace uso de notación no convencional, logrando que la guitarra eléctrica sea un instrumento improvisado, lo que la conecta con la tradición del blues y del rock, enmarcándola en una forma común de música de concierto, sugiere Tomaro.

Es claro que una discusión alrededor de la improvisación sería realmente extensa y no está dentro de los objetivos de este texto ni del escrito de Robert Tomaro, sin embargo, no se puede desconocer que dentro de la práctica de la música académica contemporánea existe también algo denominado improvisación. Por lo tanto, es justo aclarar qué elementos idiomáticos crean el vínculo del concepto de improvisación dentro de la obra de Sharp con las prácticas del blues y el rock.

En este texto también se afirma que Vicious Cycle es una pieza que no retrata la intrusión de la guitarra eléctrica en la música de concierto, sino que adopta el ambiente natural del instrumento ajustado en una forma tradicional de música de concierto. Con esto en mente, podría afirmar que desde String Trio (1960) hasta Vicious Cycle (1984) son 24 años en los que la música contemporánea estadounidense para guitarra eléctrica estaría basada en retratos de otras músicas 
tradicionales. A pesar de esto, ¿se podría afirmar que la música contemporánea ha logrado incorporar la guitarra eléctrica en sus diferentes estéticas sin la necesidad de hacer referencias a otros estilos musicales, incluso durante el periodo que comprende el análisis de Tomaro? Por otro lado, las incógnitas respecto a la sonoridad de la guitarra eléctrica y las técnicas no encuentran respuesta dentro del análisis del autor.

Luego de este análisis se podría replantear una de las ideas expuestas por Tomaro, que, por cierto, es la que más cercanía tiene con el objetivo del presente escrito: el desarrollo interpretativo en la música de concierto estadounidense al que se refiere el autor sería en realidad el desarrollo de la práctica interpretativa de la guitarra eléctrica en la música estadounidense y cómo esto se refleja en la música académica contemporánea. Este se convierte en un punto interesante en el cual profundizar, teniendo en cuenta que muchos intérpretes de este instrumento nos acercamos a esta estética musical alterna con conocimientos previos de una u otra música popular estadounidense que sí hacen parte de los programas académicos de interpretación de la guitarra eléctrica.

Posteriormente, este texto expone diferentes factores que ejemplifican por qué la guitarra es un símbolo de la cultura estadounidense, discusión que no es fundamental para el entendimiento de la guitarra eléctrica dentro de la música académica contemporánea. Por esto, cierro el análisis de este texto con una última reflexión lanzada por Tomaro en la que afirma que la guitarra eléctrica cumple el papel de aguijón musical para el statu quo, y, por lo tanto, como instrumento representativo de la juventud contemporánea, su verdadero poder es mitigado al incluirla en instituciones tales como las salas de concierto. Sin embargo, con la evidencia existente, ¿podrían las salas de concierto y la música académica ser testigos y promotores de un continuo desarrollo de la guitarra eléctrica?

A través de los años, la guitarra eléctrica se ha ganado un puesto importante entre los círculos musicales académicos y esto no se ve únicamente reflejado en la creación de programas académicos de educación superior o en la enorme cantidad de material de estudio existente dentro de diferentes géneros musicales, sino también en las investigaciones que buscan demostrar la diversidad de opciones compositivas e interpretativas de este instrumento, incluso dentro del campo de la música académica contemporánea específicamente. Guitarra eléctrica y creación musical contemporánea escrito por Benoît Courribet y Santiago Quintans es una de estas.
Pese a que este texto se enfoca en la composición para la guitarra eléctrica, existen algunos puntos que vale la pena resaltar, los cuales son evidencia del tratamiento de la guitarra eléctrica dentro de la música contemporánea de concierto. Uno de los objetivos más importantes es demostrar que la guitarra eléctrica, gracias a su estructura particular, un bagaje sonoro variado y una identidad flexible, ofrece posibilidades todavía inexploradas para la creación musical. Este argumento se refuerza con la afirmación: “la guitarra eléctrica, la cual desde su evolución está intrínsecamente unida a la música popular, todavía no ha sufrido una 'renovación modernista' en el sentido de un trabajo de composición exhaustivo que explote todas sus posibilidades de expresión" (Courribet y Quintans, 2010).

Si bien en el texto no se desconoce el trabajo compositivo de artistas como Hugues Dufourt, Tristan Murail, David Torn, Elliot Sharp, Glenn Branca y Keith Rowe, declarar que no hay un trabajo de composición profundo dedicado a la guitarra eléctrica puede llegar a ser una conclusión apresurada, si no se tiene en cuenta el vasto repertorio existente, incluso enfocándonos únicamente en la música electroacústica, como lo sugieren los autores.

Desde la perspectiva tanto del compositor como del intérprete es enriquecedora la idea de guitarra como network esbozada en el texto. Básicamente, se analiza la capacidad de la guitarra eléctrica de ser conectada a diferentes medios electrónicos, resaltando la importancia del amplificador y los múltiples puntos entre la guitarra eléctrica y el sonido amplificado en los cuales el sonido puede ser modificado. Para el instrumentista que incursiona en el mundo de la interpretación de la música académica contemporánea estas ideas ofrecen luces sobre las diferentes herramientas que desde la perspectiva de otros estilos no son obvias y que para el género se constituyen como la gran cantidad de elementos posibles (incluyendo el espacio, la partitura, etc.) que pueden enriquecer la interpretación de una obra.

El escrito aborda la composición realizada por Santiago Quintans 5 fragments pour GE, la cual usa los elementos expuestos en la primera parte. De manera global vale la pena detenerse en la descripción de la figura del intérprete dentro de la música popular:

Habiéndose popularizado como instrumento en músicas de orientación "popular" (...) como el rock, el pop y el Jazz, el universo técnico/estético de la GE se ha constituido a partir de las diversas 


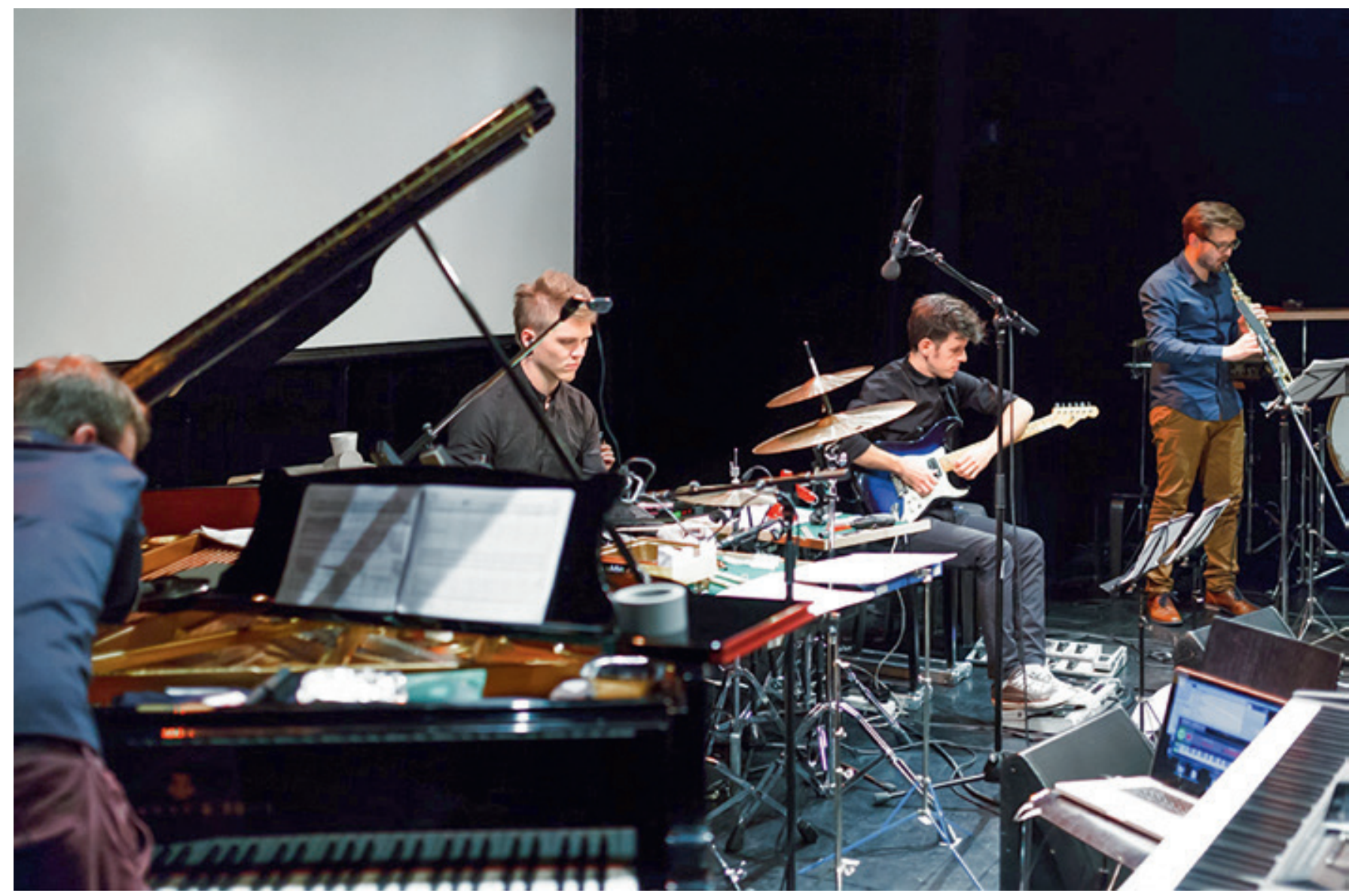

Ensemble Nikel. Foto: Markus Sepperer. En la foto de izquierda a derecha: Antoine Françoise, Brian Archinal, Yaron Deutsch, Patrick Stadler.

aportaciones de los intérpretes que participan a la evolución de estos estilos. Así, podemos decir de forma general que el desarrollo musical de la guitarra eléctrica se concentra en varios núcleos estéticos de tradición evolutiva. A pesar de la existencia de excelente material pedagógico escrito, (...) la evolución estética de la guitarra se construye entorno a la figura del creador/intérprete: instrumentistas cuya importancia se deriva no solo de su facilidad con el instrumento, sino también de las contribuciones técnicas y, sobre todo, estéticas a un repertorio estilístico concreto (...). (Courriber y Quintans, 2010)

Para los autores, esto presenta diferentes limitaciones creativas que no abordaré por referirse específicamente a la composición. Sin embargo, se debe tener en cuenta que dentro de la música académica contemporánea la figura de compositor y la del intérprete se encuentran normalmente separadas, lo que aportaría diferentes perspectivas, así como también la posibilidad de múltiples interpretaciones de una misma pieza., dando así un rango amplio de libertad creativa para el intérprete.
En relación con la pieza resumo algunos aspectos. Dentro del network, la notación musical se constituye como el primer elemento, el cual se divide entre tradicional y no convencional; adicionalmente, se toma como importante el ámbito físico, que es la relación entre intérprete e instrumento; por otro lado, la improvisación adquiere un papel importante, ya que aporta a la concepción global de la obra, a través de elementos tomados de la misma: tanto la electrónica a través de pedales y la electrónica por computador constituyen otro elemento que enriquece este network. Por último, se hace mención al uso de objetos para la preparación de la guitarra y se cita el uso de técnicas instrumentales contemporáneas, que en realidad se recogen de su uso en la música popular, como el tapping.

Este es un ejemplo de las muchas posibilidades creativas que puede encontrar un intérprete dentro de la música de concierto contemporánea. Adicionalmente, y como se destaca en las conclusiones del escrito analizado, la guitarra eléctrica se convierte en un instrumento que presenta infinitas posibilidades para los compositores de hoy, gracias a las capacidades de 
transformación en cada uno de los puntos que puedan conformar su network.

\section{Sheer Pluck Data Base}

Hasta el momento, la literatura relacionada con la guitarra eléctrica dentro de la música contemporánea hace un fuerte énfasis en diferentes posibilidades y retos en la composición para este instrumento. A pesar de ello, se ha hecho evidente que dentro de estos textos se omite de una manera u otra el extenso repertorio creado alrededor del mundo. Por este motivo se hace importante que centre mi atención en sheerpluck.de, base de datos en línea de música contemporánea para guitarra. Este sitio web, diseñado para proveer información no solo para instrumentistas, sino también para investigadores, estudiantes y todo aquel que pueda estar interesado por el desarrollo de la música contemporánea para guitarra en el siglo XX y XXI, es el resultado de la unión de dos ideales afines. El primero de ellos fue concebido en el 2002 como una página web creada por Klaus Heim, Contemporary Guitar Composers, que referenciaba diversos autores de música para guitarra enmarcada en el ya mencionado género. Todo esto con el propósito de facilitar una vista general del creciente repertorio. El segundo de ellos, un proyecto del guitarrista Seth Josel orientado a la recopilación de información acerca de música contemporánea para guitarra eléctrica.

Sheer Pluck Data Base actualmente recoge 67.403 piezas, de las cuales 47.338 son visibles al público en general y que, además, aproximadamente 5.113 de ellas fueron compuestas por compositores alrededor del mundo que incluyeron la guitarra eléctrica dentro de su instrumentación. La información contenida en esta base de datos implicó una búsqueda exhaustiva en todos y cada uno de los registros consolidados en esta página, lo que permitió el acceso a los sitios web de algunos compositores, información de contacto, partituras, audios, etc.

Teniendo en cuenta que el periodo que comprende este análisis empieza en la segunda mitad del siglo $X X$, es decir 1950, es realmente significativo el hecho de que dentro de las obras que se registran en esta fuente de información durante la primera década (1950 a 1959), se encuentran 32 composiciones realizadas por compositores con nacionalidades italiana, francesa, argentina, alemana, estadounidense, japonesa, entre otras. Siendo aún más significativo que las composiciones Concerto carioca No.1 y Studi per il 'Processo' di Kafka de los compositores Radamés Gnattali (Brasil, 1906 - 1988) y Bruno Maderna (Italia, 1920 - 1973), respectivamente, datan del año 1950. Ambas piezas enmarcan la guitarra dentro del contexto de orquesta. Posteriormente el número de piezas crece de manera significativa, siendo el siglo XXI (años entre el 2000 y 2016) una época importante de crecimiento de este repertorio, ya que durante estos 16 años se registran cerca de 2.764 obras sin contar, naturalmente, las que puedan quedar fuera de esta base de datos.

Lo anterior es clara evidencia del desarrollo de la guitarra eléctrica en la música académica contemporánea, que va paralelo a su creación y evolución tecnológica, como también a su participación y transformación en diferentes músicas populares. Es innegable la influencia que ejercen estas últimas en la creación académica contemporánea; sin embargo, no existe un análisis de los aportes de la música de vanguardia en sí misma, más allá de la comparación o apropiación de músicas populares.

\section{Lo que suena}

Si bien el repertorio existente, que incluye reconocidos compositores como Luciano Berio, Kalrheinz Stockhausen, Morton Feldman, Earle Brown, Christian Wolff, Glenn Branca, entre muchos otros, y las preocupaciones de diferentes autores, crean un panorama amplio de análisis que legitimaría la guitarra eléctrica dentro de la música académica contemporánea, esto aparentemente no es suficiente. Como se afirma en el texto The Electric Guitar in Contemporary Art Music, escrito por Zane Mackie Banks, la participación del intérprete es de vital importancia.

Mientras los orígenes de la guitarra acústica permanecían en tradiciones folclóricas y regionales, esta adquiría asociaciones culturales de las cuales cierta parte de la élite de la música de arte no quería ocuparse. La guitarra clásica luchó por una amplia aceptación en la comunidad de la música de arte durante la primera mitad del siglo XX. Fueron los incansables esfuerzos de Andrés Segovia (1893 - 1987), Julian Bream (1933) y John Williams (1941) los que elevaron el perfil de la guitarra clásica, presentándola como instrumento de concierto virtuoso y legítimo. (Mackie, 2013: 4).

Con esto en mente, es necesario no solo realizar un trabajo constante y serio, abriendo espacios para la interpretación y presentación de este repertorio, sino que también, en este caso, vale la pena destacar el 


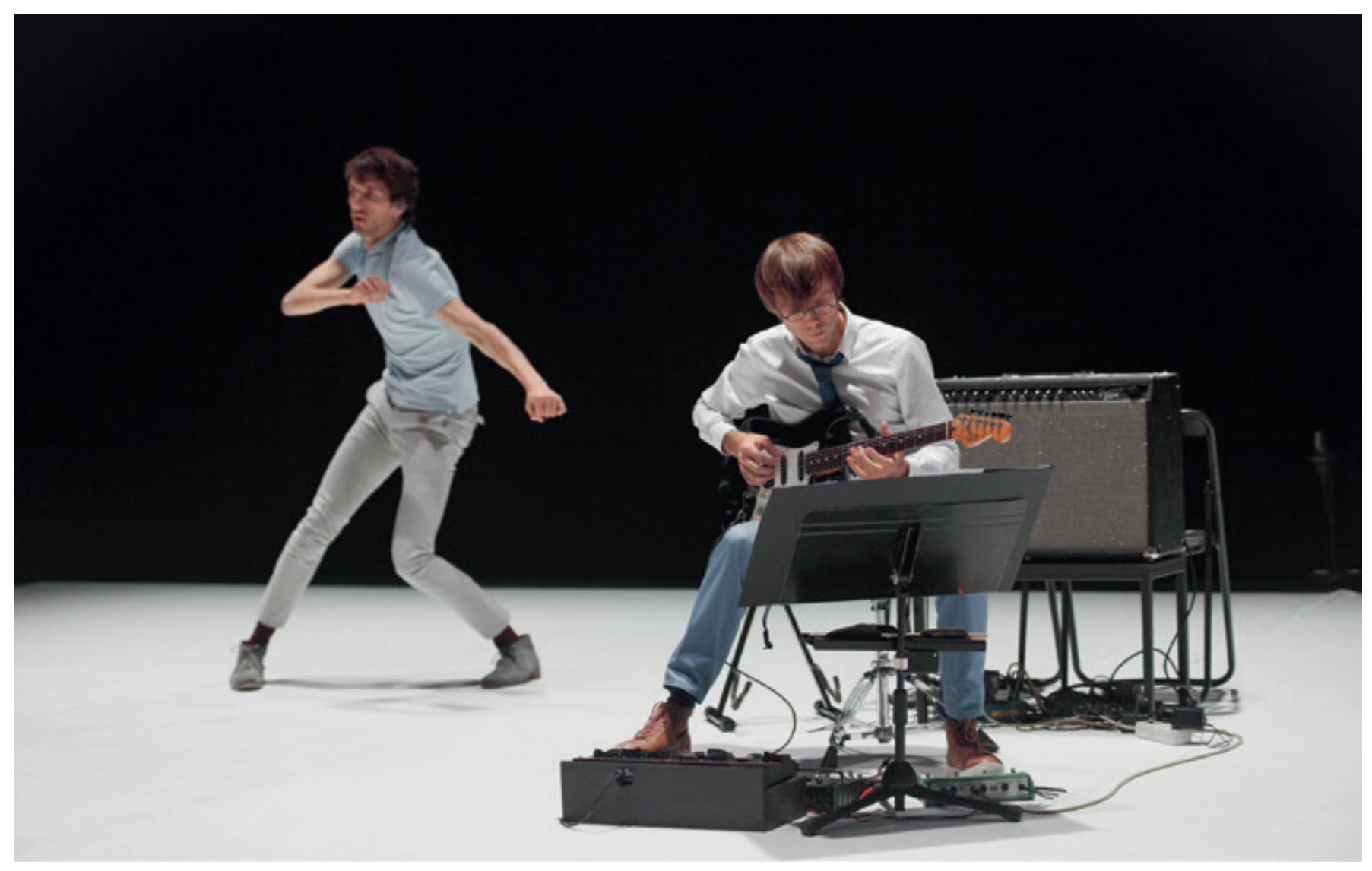

ICTUS Ensemble. En la foto: Tom Pauwels (Guitarrista). Imagen cortesía del artista.

trabajo realizado por algunos guitarristas eléctricos y ensambles que cuentan con la guitarra eléctrica como miembro constante de su instrumentación y para quienes su foco de trabajo es la interpretación de música académica contemporánea. Esto implica dejar de lado grandes guitarristas que son cercanos a esta estética musical, pero desde la perspectiva de la improvisación libre, para enfocar mi atención en la interpretación de repertorio para o con guitarra eléctrica.

En primer lugar, centro mi atención en una de las más reconocidas organizaciones promotoras de nueva música basada en Nueva York, Bang on a Can. Entre sus múltiples programas, formado en el año 1992, se encuentra el ensamble Bang on a Can All Stars, el cual entre sus miembros cuenta con Mark Stewart como intérprete de la guitarra eléctrica. Dentro de los proyectos realizados por esta agrupación se encuentran múltiples trabajos discográficos, colaboraciones en composiciones y presentaciones en vivo, todo esto con artistas como Steve Reich, Philip Glass, Thurston Moore, entre otros. Es notable que, además, esta organización anualmente organiza el Bang on a Can, Summer Music Festival. Para esto, se convocan compositores e intérpretes interesados en profundizar en la música académica contemporánea, teniendo la posibilidad de asistir a diferentes talleres especializados en distintas áreas.

En esta misma ciudad se funda en el año 2007 el cuarteto de guitarras eléctricas Dither Quartet, conformado por los guitarristas Taylor Levine, Joshua Lopes, James Moore y Gyan Riley, quienes dedican su actividad a la interpretación de repertorio experimental y a la improvisación, presentando su propuesta musical en diferentes espacios al rededor del mundo. Su trabajo abarca un amplio rango de artistas entre los que se destacan Eve Beglarian, Fred Frith, David Lang, Phill Niblock, Larry Polansky, Lee Ranaldo, Elliott Sharp, Lois V. Vierk, y John Zorn. Adicionalmente, este cuarteto produce anualmente el festival Extravaganza de arte y música creativa.

Por otro lado, esta vez con sede en Bélgica, se destacan las actividades de dos ensambles. Al primero al que me referiré es ICTUS, conformado por 20 músicos, entre ellos el guitarrista Tom Pauwels quien enfoca su interpretación a la guitarra eléctrica y acústica por igual. Esta agrupación dedicada de forma muy activa mundialmente a la música de concierto contemporánea desde 1994, promueve, especialmente en Bruselas, el acercamiento de nuevas audiencias no especializadas 


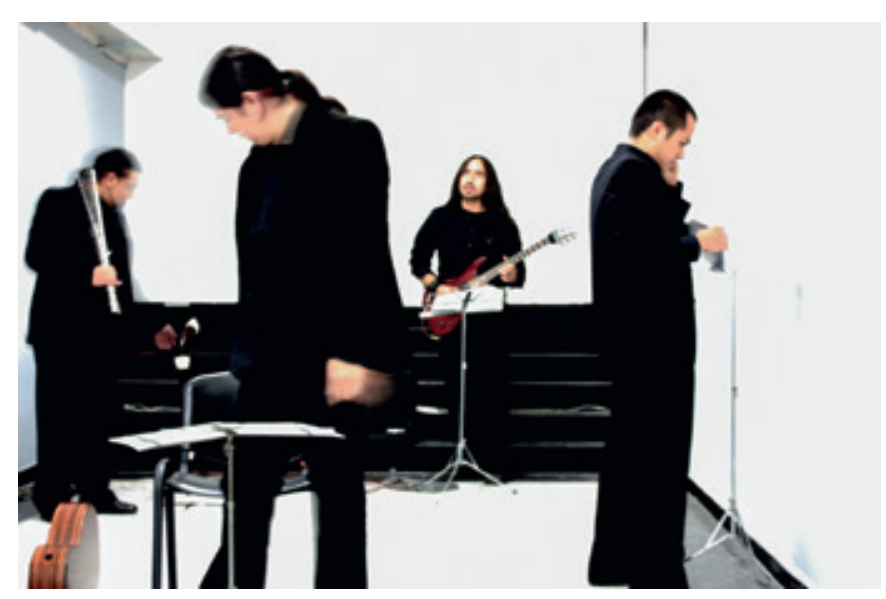

Grupo Otraparte. Foto: Grupo Otraparte. En la foto de izquierda a derecha: Leonardo Peña, Juan Manuel Bernal, Carlos Romero, Carlos Díaz Borbón.

juntando esfuerzos con compañías de teatro y danza. Adicionalmente, en colaboración con la facultad de música del University College Ghent y la agrupación de Ghent Spectra Ensemble ofrecen estudios de maestría avanzada enfocada en el perfeccionamiento de la interpretación de música académica contemporánea solista y en ensamble de cámara. El segundo ensamble belga que toma protagonismo es Zwern. Fundado en el 2007, es un cuarteto de guitarras eléctricas conformado por los guitarristas eléctricos Johannes Westendorp, Bruno Nelissen, Toon Callier y Kobe van Cauwenberghe. Entre sus múltiples proyectos enmarcados en la música experimental, se destaca Zwern ft. Fred Frith concierto junto al guitarrista, improvisador y compositor británico Fred Frith.

Dirigido y fundado en el año 2006 por el guitarrista eléctrico Yaron Deutsch se encuentra el ensamble Nikel, con base en la ciudad de Tel Aviv. Este cuarteto de guitarra eléctrica, piano, saxofón y percusión, ha actuado como invitado en diferentes festivales dentro del marco de la música de vanguardia como los organizados por el Bang on a Can. También, promueve la composición de nuevo repertorio trabajando en conjunto con compositores como Marco Momi, Helmut Oehring, Stefan Prins, entre otros. Al igual que algunos de los ensambles anteriormente nombrados, Nikel organiza el International Festival and Summer Course for New Music Performance and Composition - Tel Aviv, Israel, dedicado a la composición e interpretación de nueva música.

Indudablemente, tomar parte en la interpretación de obras con instrumentaciones variadas y numerosas, o por lo menos con más de un instrumento, ofrece un sin número de experiencias que enriquecen el quehacer del intérprete, sin embargo. no es estrictamente necesario enmarcar la guitarra eléctrica únicamente en el contexto de ensamble. El trabajo de guitarristas como Wiek Hijmans y Seth Josel son evidencia de este hecho, siendo reconocidos en mayor medida por su trabajo como guitarristas, más allá que por hacer parte de una agrupación específica.

De origen neerlandés, Hijmans se posiciona como uno de los guitarristas eléctricos más importantes de la escena contemporánea, con un prolífico recorrido artístico en el que se encuentran recitales como solista, presentaciones con ensamble, diferentes proyectos con múltiples agrupaciones y clases maestras en diferentes instituciones, entre otros.

Por otro lado, Seth Josel, quien reside en Berlín, pero es de origen estadounidense, y que de manera anticipada mencioné como cofundador de Sheer Pluck Data Base, presenta también una florida carrera como guitarrista, no solo solista sino también haciendo parte de diferentes ensambles. Ha realizado el estreno de múltiples comisiones $y$, adicionalmente, ha colaborado con compositores de renombre mundial. Aunado a esto, Josel ha realizado varias producciones discográficas $y$, junto a Hijmans, cofundó el ensamble de guitarras eléctricas Catch.

\section{En Colombia}

Con el tiempo la guitarra eléctrica ha tenido un papel cada vez más activo en nuestro país, convirtiéndose incluso en un instrumento de alta demanda educativa. Directamente en el ámbito de la música académica contemporánea, han surgido algunos ensambles que ponen la guitarra eléctrica como instrumento principal en sus interpretaciones, como es el caso del grupo Otraparte y el recientemente fundado Ensamble Kling. En este apartado debo destacar que parte del material recogido a lo largo de esta investigación fue interpretado en mi recital de último semestre del énfasis de interpretación de la guitarra eléctrica; esto, pensando (más allá que en mi interés por la música académica contemporánea) en demostrar que el estudio de este repertorio puede desarrollar y perfeccionar las destrezas que se necesitan como intérprete.

Por otro lado, es también creciente el interés de algunos compositores por asumir el reto de escribir para este instrumento. Pese a que no podría afirmar que en Colombia la composición de música contemporánea 
para guitarra eléctrica sea prolífica o que existan compositores dedicados casi exclusivamente a ello, sí podría destacar algunas piezas que engrosan el repertorio del instrumento. Como la obra más antigua que hasta el momento puedo referenciar dentro del repertorio colombiano esta Carceris Tonalis (1995), del compositor Rodolfo Acosta (Col., 1970), escrita para grupo de cámara con guitarra eléctrica solista y objetos amplificados también solistas. Esta pieza emplaza la guitarra eléctrica en un rico panorama tímbrico, no solo por los instrumentos que conforman el ensamble, sino también por la gama de recursos usados para tañer la guitarra como uso de barra de vibrato, plectro, arco de contrabajo, slide metálico, guitarra preparada con fósforos, taladro eléctrico, aunado a uso de wah y distorsión. Carceris Tonalis presenta un tratamiento ampliamente libre de la guitarra eléctrica, sin especificar afinación exacta; se hace uso de notación no convencional y proporcional, se detallan los momentos en los que se requiere uno u otro timbre, pero únicamente sugiriendo mediante gráficos la evolución de los sonidos.

Posterior a esta pieza se podría afirmar que el grueso de composiciones, que probablemente no supera la decena y media, nacen ya iniciado el siglo XXI. En las dos siguientes piezas que referenciaré, he tenido el privilegio de acompañar el proceso compositivo y, posteriormente, realizar el estreno de las obras. La primera a la que me refiero es Tatuaje (2013) para violonchelo, guitarra eléctrica, piano y voz, escrita por la compositora Melissa Vargas (Col., 1980). La obra exhibe una guitarra sobria, exigiendo únicamente para algunos pasajes el uso de eBow y, como elementos adicionales al sonido del instrumento, el uso de una reverberación y un delay. Estos últimos son notados en la partitura como un instrumento adicional justo debajo del sistema de la guitarra, con el fin de marcar las diferentes transiciones que presentan dichos efectos y que deben ser manipulados por el guitarrista mismo. Además de estar escrita con notación convencional, la sección de improvisación, la cual es cercana a la estética de la improvisación libre es, en realidad, una improvisación con ciertos parámetros determinados, lo que permite que el intérprete que no tiene experiencia en este tipo de sonoridades explore de una manera guiada esta corriente de improvisación.

...la fragilidad de mis sentidos cuando estás cerca, y de ese contacto quiero cada vez más... (2011) para guitarra eléctrica y electrónica en vivo del compositor Maicol Cadena (Col., 1988) es una pieza que involucra al instrumentista en la interpretación, no solo de la guitarra, sino

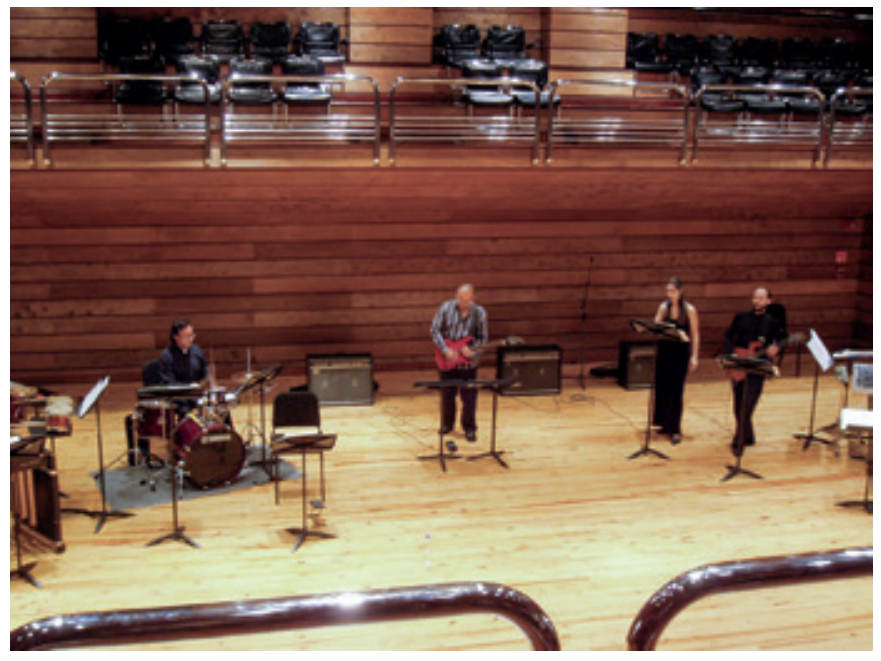

Concierto Ensamble CG durante el XVI Festival Latinoamericano de Música en 2010, Caracas, Venezuela, 2010. Foto: Ensamble CG. En la foto de izquierda a derecha: Eduardo Caicedo, Rodolfo Acosta, Beatriz Elena Martínez, Guillermo Bocanegra

de sus pedales de efectos también. Para esta composición es únicamente necesario el uso de un pedal de delay con ciertas capacidades de tempo. Especial en esta pieza son también las secciones de improvisación que no se realizan con la guitarra, sino que con el delay mismo, siguiendo ciertos parámetros básicos sugeridos por el compositor. Este tipo de hechos expande la concepción del instrumento, haciendo parte de él toda la red de artefactos que existen entre la guitarra y el sonido amplificado, hecho que encuentra respaldo en los argumentos antes tratados en los antecedentes del presente escrito.

Por último, en el año 2011 el compositor Luis Fernando Sánchez (Col., 1982) fue ganador de la Beca de Creación: Música Contemporánea, otorgada por el Ministerio de Cultura de Colombia, para realizar la composición En la cercanía, en cada centímetro de piel... en cada poro (2011-2012), para guitarra eléctrica, electrónica en vivo (interfaz tipo mesa interactiva) y electrónica en medio fijo. Para el guitarrista eléctrico no existen grandes exigencias más que ciertas recomendaciones flexibles de equipo, sin embargo, haciéndose muy importante el tener la comodidad para tocar sin plectro (fingerstyle) o con plectro y dedos (hybrid picking), lo que ya es un reto, teniendo en cuenta que este tipo de técnicas no se cultivan de manera especial en la academia. La pieza se encuentra escrita con notación convencional con algunas aclaraciones pertinentes para ciertos pasajes. Esta pieza es muestra de la importancia que poco a poco va adquiriendo la guitarra eléctrica en Colombia y, aún más importante, dentro de la creación musical contemporánea. 


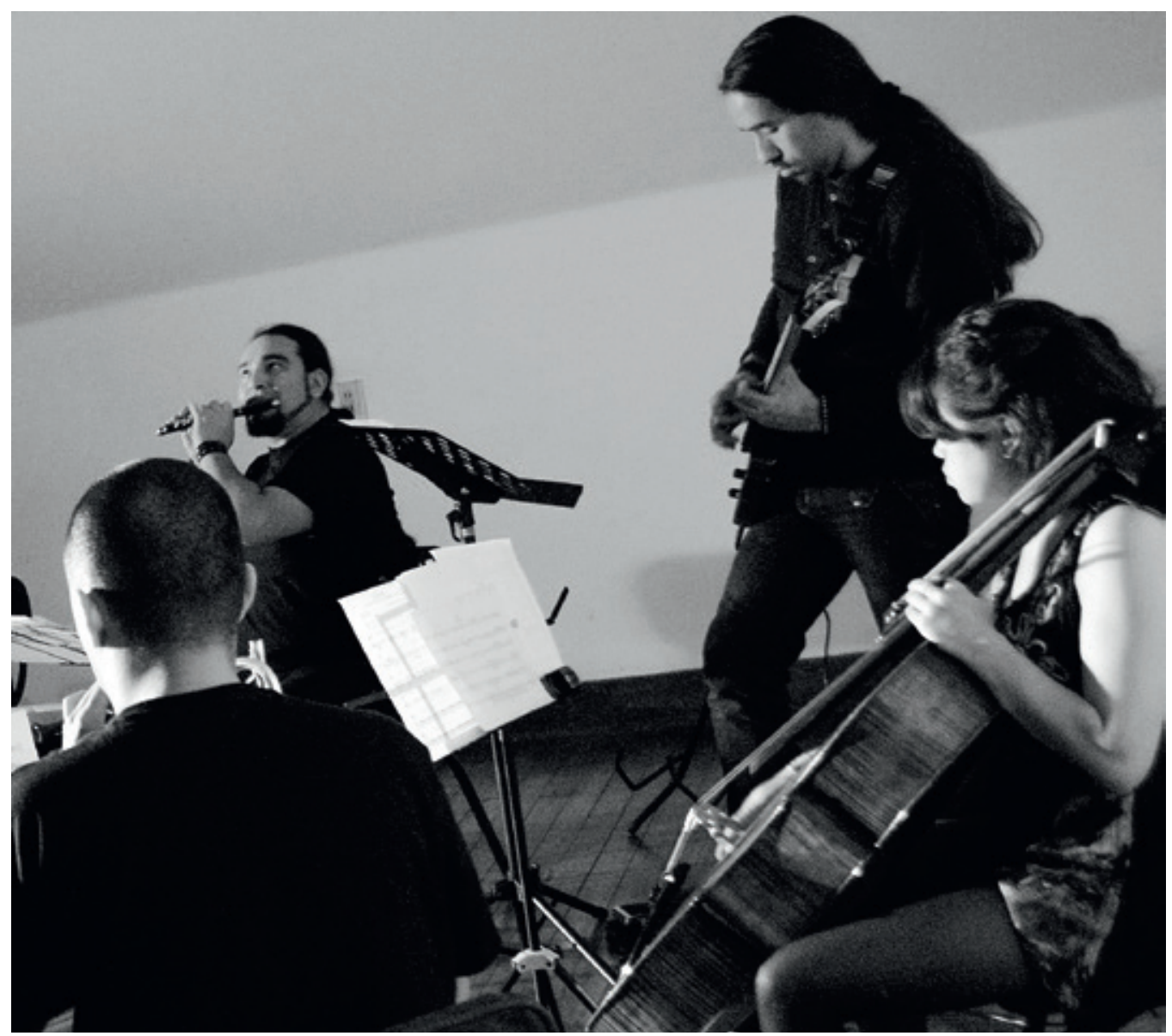

Grupo Otraparte. Foto: Grupo Otraparte. En la foto de izquierda a derecha: Carlos Díaz Borbón, Leonardo Peña, Carlos Romero, Carolina Moreno.

\section{Conclusiones}

Es indudable que la guitarra eléctrica ha tenido una vida paralela a la música popular y que se ha gestado en las salas de concierto y academias, que seguramente ha desarrollado y explotado mucho de su potencial musical y que es muestra de diferentes perspectivas compositivas de música académica contemporánea alrededor del mundo.

La música contemporánea ofrece escenarios instrumentales enriquecidos por diferentes timbres con los que interactúa el instrumento, como los ensambles de cámara, su participación con orquestas y el trabajo con electrónica. Razón por la cual el intérprete se ve en la necesidad de superar los retos que esto implica y que, con un constante análisis y reflexión de los materiales existentes hasta ahora sobre el tema, encontrará los criterios apropiados para iniciar y profundizar en la interpretación de este repertorio.
Se ha logrado evidenciar que, pese a la existencia de un par de investigaciones previas que esbozan el ámbito de la guitarra eléctrica contemporánea, estas no son prueba suficiente de los vastos frutos de la composición para el instrumento en este género. Pese a lo anterior, sí existe interés y ánimo por parte de músicos, en su gran mayoría compositores, en brindar herramientas para la evolución de este campo, debido a que ven el potencial musical que este instrumento les ofrece.

Por último, este tipo de preocupaciones son el inicio y base sobre la cual se podrían edificar múltiples proyectos, que incluso antes de su publicación en el caso de estos resultados de investigación, han sido de apoyo para estudiantes, docentes y agrupaciones musicales que encuentran una valiosa base para innovar en su quehacer. Naturalmente, la invitación final es a que este tipo de iniciativas sean el detonante para el trabajo profundo en este campo, dando cada día más relevancia a la guitarra eléctrica en la música académica contemporánea. 


\section{Referencias}

Bang On A Can, Summer Music Festival [en línea]. (s.f). Disponible en: http://bangonacan.org/summer_festival. [Consultado 20 de octubre, 2014].

Circulo Colombiano de Música Contemporánea. (2010). Estatutos [en línea]. Disponible en: http://www.ccmc. com.co/admin/ccmc/estatutos_ccmc.pdf. [Consultado 20 de octubre, 2014].

Courribet, Benoît y Santiago Quintans (2010). Guitarra eléctrica y creación musical contemporánea. Espacio Sonoro, revista quatrimesta de musica contemporanéa (1) [en línea]. Disponible en: http://www.tallersonoro. com/anterioresES/21/Articulo2.htm. [Consultado 20 de octubre, 2014].

Courribet, Benoît y Santiago Quintans (2010). “Guitarra eléctrica y creación musical contemporánea", en: Espacio Sonoro, revista quatrimesta de musica contemporanéa (2) [en línea]. Disponible en: http://www. tallersonoro.com/anterioresES/22/Articuloguitarra.htm. [Consultado 20 de octubre, 2014].

Heim, Klaus y Seth Josel (2002). Sheer Pluck, Database of contemporary guitar music [en línea]. Disponible en: http://www.sheerpluck.de. [Consultado 20 de octubre, 2014].
Ictus. (s.f). Academy (School of Arts Ghent | Ictus | Spectra). Disponible en: http://www.ictus.be/projects/ academy. [Consultado 20 de octubre, 2014].

Internationales Musikinstitut Darmstadt. (s.f). Internationale Ferienkurse für Neue Musik [en línea]. Disponible en: http://www.internationales-musikinstitut. de/ferienkurse.html. [Consultado 20 de octubre, 2014].

Mackie, Zane (2013). The Electric Guitar in Contemporary Art Music. Sydney Conservatory of Music, Sydney University.

Quintans, Santiago (2010). «La guitare électrique comme moyen de création au sein d'un réseau d'outils compositionnels ». Revue Appareil [en línea]. Disponible en: http://appareil.revues.org/1021. [Consultado 20 de octubre, 2014].

Tomaro, Robert (1994). “Contemporary Compositional Techniques for the Electric Guitar in United States Concert Music". Journal of New Music Research, 23:4, 349-367.

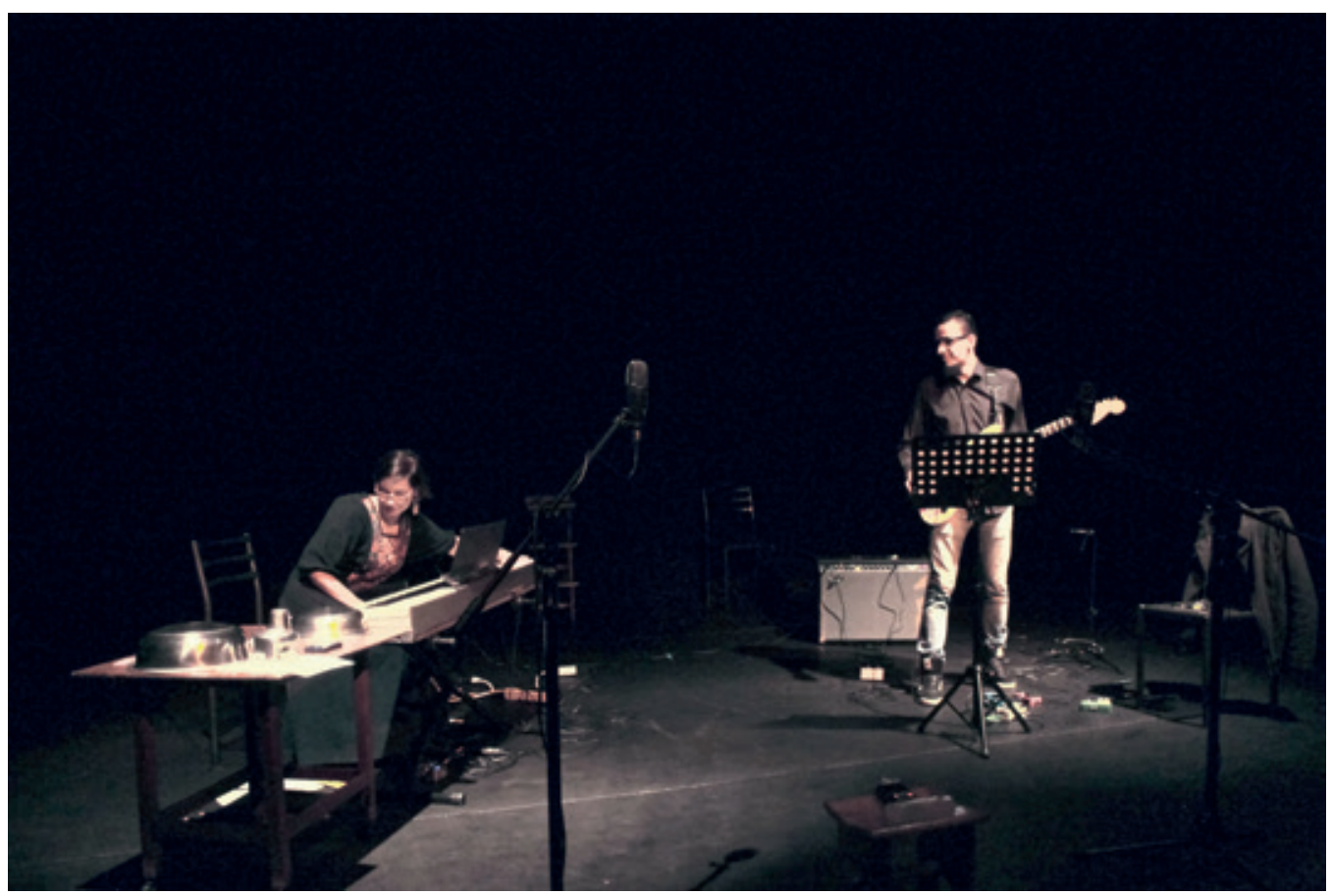

Concierto Ensamble Kling, Teatro Libélula Dorada, 6 de Septiembre de 2016 Foto: Esteven Niño. En la foto de izquierda a derecha: Melissa Vargas, Johan León. 